\title{
Labor Commitment in Social Workers Before Covid-19
}

\author{
Cruz García Lirios ${ }^{1 *}$, José Marcos Bustos Aguayo² ${ }^{2}$ Margarita Juárez Nájera ${ }^{3}$ and Francisco \\ Ruben Sandoval Vazquez ${ }^{4}$
}

${ }^{1}$ Department of Social Work, UAEMEX, Mexico

${ }^{2}$ Department of Psychology, UNAM, Mexico

${ }^{3}$ Department of Energy, UAM, Mexico

${ }^{4}$ Department of Political Science, UAEM, Mexico

*Corresponding author: Cruz García Lirios, Department of Social Work, UAEMEX, Mexico

\section{ARTICLE INFO \\ Received: 幽 October 15, 2020 \\ Published: 幽 November 04, 2020}

Citation: Cruz García Lirios, José Marcos Bustos Aguayo, Margarita Juárez Nájera, Francisco Ruben Sandoval Vazquez. Labor Commitment in Social Workers Before Covid-19. Biomed J Sci \& Tech Res 31(4)2020. BJSTR. MS.ID.005134.

\section{ABSTRACT}

Social work is involved in public policy and social programs that target vulnerable, marginalized or excluded, it is necessary to analyze its commitment considering its relation to the management for the treatment of illness and rehabilitation of health sector institutions. In this regard, the objective of this study was to establish the reliability and validity of an instrument for measuring work commitment in the health sector. For this purpose, we used a nonrandom sample of 100 social work professionals with experience in implementing social programs and monitoring of institutional strategies for health promotion. Were established normality, reliability and validity on the scale that measured labor commitment. Structural model was established in which reflective commitment to the institution was positively related to work engagement. The residual fit indices and corroborated the hypothesis of multidimensionality of work commitment.

Keywords: Public Policy; Health Sector; Social Work; Occupational Commitment

\section{Introduction}

At the time this work is written down, the pandemic caused by the SARS-CoV-2 coronavirus and COVID-19 have infected 15 million, sickened 7 million and killed 700,000 globally. In Mexico, 400 thousand infected, 100 sick and 40 deaths are registered. Because governments have implemented their mitigation policies from a central health authority, constitutional laws prevent a strict and permissible confinement only before the declaration of a State of exception, contagions have exceeded the institutional capacity of the public health service [1]. In the case of Mexico, the health culture that inhibits disease prevention, as well as resistance to medical care until a critical and irreversible stage, coupled with the discretionary and non-selective recruitment of 50,000 health professionals, hospitals without equipment and the lack of ambulances have led to the deaths of 40,000 and at least 80,000 deaths are projected. In this complex scenario, work commitment understood as provisions against or in favor of health policies, public assistance service, self-care and social support have been observed as a complex and multidimensional variable that explains the exposure to risks by health workers, as well as the prevalence to assume more risks expecting minimum benefits or acknowledgments [2].

Precisely, the objective of this work was to establish the dimensions of work commitment, considering the scenario of risks associated with the health crisis (COVID-19) and the economic crisis (recession). The hypothesis that guides the present study indicates a prevalence of multidimensional commitment in a situation of risk or health contingency [3]. It is a construct that explains at least four axes of discussion and research on the subject once health actors interact with political agents and social sectors [4]. In this way, the commitment is diversified according to the demands of the environment, institutional resources, the capacities of professionals and the dispositions of users of the public service [5]. The diagnosis will allow anticipating the responses of health professionals in general and social workers in the face of a probable scenario of risks, contingencies and threats to their health and integrity. In this way, in the first section the theoretical and conceptual frameworks that explain the labor commitment are exposed. A second section includes studies related to the dimensions of work commitment in 
order to be able to select those for modeling in a third moment. The methodological aspects are included, as well as the results and the discussion with the state of the question in order to be able to make a final reflection on the subject before COVID-19.

\section{Theory of Labor Commitment}

In the framework of the pandemic mitigation policies, scenarios of confinement and violence prevail among the parties involved, as well as between the rulers and the governed attributable to risk management, management and communication [6]. In such a situation, health professionals develop expectations before their authorities and users of public health services, as well as before the infrastructure of their work areas. In this sense, the theoretical and conceptual frameworks that explain their degree of commitment refer to their dispositions, intentions, and actions in the face of scarcity, shortages, unhealthiness, and famine. In this way, the explanatory theories of the organizational and personal situation of health professionals were designed and consolidated in the $1970 \mathrm{~s}$ [7]. These are the Theory of Reasoned Action, the Theory of Setting the Agenda, the Theory of Prospective Decisions and the Theory of Amplification of Risks. In a context where health institutions and organizations were considered as balanced environments of demands and resources, the Theory of Reasoned Action contributed to the state of the question by relating the beliefs of abundance or scarcity with the provisions against or in favor of their performance [8]. Occupational. This is the case of health professionals who, by interacting with their environment, developed self-management skills that earned them the formation of associations such as "Doctors without Borders" or the "International Red Cross" itself. From this theoretical approach, engagement was the result of a deliberate, planned, and systematic process of biomedical rather than social or organizational decisions for the well-being of health professionals.

In that same decade of the sixties, the media reached the status of fourth power by highlighting the political and institutional blunders in corruption scandals associated with health policies in the face of the impact of natural disasters, pollution from multinationals or nuclear accidents [3]. These are environmental organizations that questioned the state's programs and strategies in the face of the extinction of animal and plant species. Consequently, this theoretical approach considered commitment as the product of establishing axes and topics for discussion on the public agenda derived from the political agenda and this in turn influenced by the scientific and investigative health agenda.

At the same time, the Theory of Prospective Decisions focused on the study of health promotion, the prevention of accidents or diseases, as well as the self-care and adherence to treatment of users of the public health care service [9]. This theoretical corpus linked decisions in risky situations with expectations of high costs and maximum benefits to explain risky behaviors in the face of the emergency of sexually transmitted diseases. Lastly, given the increase in cases of disease in developing countries, the Theory of Risk Amplification addressed the problem of communication and crisis management, as well as its effects on vaccination campaigns for the general population or sectorial [10]. In the case of epidemics and even more so of pandemics, the amplification of risk refers to the fact that political strategies are potentiated to their maximum expression in civil society. In this way, those who make decisions about the confinement of people acquire the commitment to generate expectations of people towards their capacity and performance, tolerance and empathy with victims and deaths.

In summary, the theoretical and conceptual approaches that explain the impact of public policies on the performance of professionals, the subjects of public service and the open population in general through the dissemination of decisions in the media have outlined sectors in order to be able to anticipate their responses to health risks and contingencies.

\section{Studies of Labor Commitment}

Work commitment is embedded in an organizational psychological system that starts from the climate and ends in satisfaction. In such a system, commitment interrelates with leadership and performance to mediate the relationship between climate and satisfaction [11]. These are assumptions according to an increase in task and human relationships that impact leadership, commitment and performance to influence an increase in life satisfaction. In other words, human capital in organizations seems to be immersed in a series of activities and personalities that lead them to assume leadership roles and dimensions of commitment that increase their performance, although such a process is rather oriented towards greater personal satisfaction, the Organization seems to intercede in such purposes. The psychology of organizations has stated that production processes are inherent in the cognitive processes of those who work in an organization [12]. In reference to organizational development, organizational psychological studies have shown that commitment is a factor in the systematization of production. In this sense, the commitment is assumed as a set of actions, roles, motives and expectations that generate a collaborative dynamic among the members of a work group or productive organization.

The model of organizational commitment presents seven cases concerning empowerment, motivation, identity, trust, ambiguity and conflict which affect the commitment through the satisfactions [13]. An increase in empowerment and motivation would lead to an increase in satisfaction and commitment. However, the reduction of ambiguity and conflict when negatively related to satisfaction affect an increase in commitment. Rather, increasing identity and confidence also influence satisfaction and commitment. If organizational commitment is determined by empowerment, motivation, identity, trust, ambiguity, and conflict through satisfaction, then commitment can be defined as the result of the 
interrelation between organizational factors of a human nature in reference to the relationship between leaders and employees [14]. In this sense, commitment is a function of personal wishes and organizational visions. It is an indicator of fairness and justice in which leaders relate to employees based on a balance between freedoms, capacities and responsibilities.

Based on such assumptions, organizational psychologists have assumed that commitment is a product rather than a permanent process of identity [15]. Those who make a commitment to their companies are considered as a product of organizational dynamics rather than as individuals with collaborative personalities and values. In this sense, the recruitment and selection of prospects is not carried out based on their individual characteristics, but rather their capacities and coping strategies in the face of the emergence of conflicts, risk and uncertainty. Organizational commitment opens the discussion around the relationship between organization and individual [16]. The influence of the first on the second seems to be corroborated with organizational psychological studies, but the commitment, as a systematization of functions and results, goes beyond the individual and the organization. The work commitment refers to a set of moral and evaluative principles characteristic of leaders who, in their eagerness to achieve their objective, firmly believe in the ideals of productivity, order and systematization of organizational functions.

In short, the commitment is a set of beliefs, attitudes and actions that reduce uncertainty and increase a propensity for the future. Increasing risk expectations would decrease motivation for work and disorganize the human relations system, affecting the performance of each member. Organizational psychological studies have established dimensions of work commitment based on their relationship with variables of an organizational nature [17]. Mainly, work commitment has been correlated with work culture, task climate life satisfaction, social recognition, transformational leadership, productive performance and entrepreneurial spirit. Psychological studies of the labor commitment have established causal relationships between and variable styles of leadership [18]. As the type of leadership intensifies, it explains the increase in work commitment. Different types of leadership affect the increase or decrease of perceptions, attitudes and actions. In this sense, the performance history affects the commitment to increase productivity in the future. Behind the labor engagement, command structures, relations task, conflict and stress or satisfaction seem to explain the increase or decrease commitment assumed by the stay labor, although attention to the customer explained labor commitment.

Coincide in relating life satisfaction with commitment [19]. Such findings suppose a continuity between socialization of the first and second order, whether in a collectivist or individualistic sense, favorable or unfavorable, the principles that guide the individual in a family group would be the same as those that guide them in a labor or productive group. In light of these results, work commitment would be the last link, at least in the workplace, of a chain of perceptions, beliefs, attitudes, decisions and actions directed from the primary group in which the individual learned the basic symbols and in whose development he never had the opportunity to question such principles that now seem to guide him in his commitment, productivity and job satisfaction. The state of the art seems to show solid evidence around the complementarity between first order socialization and second order socialization.

The dimensions of work commitment in Social Work professionals who work in public institutions but have had critical training in the exercise of their profession and have been socialized under collectivist principles where the interests of the majority prevail over the objectives personal or institutional [10]. The answer to this question is confined to organizational psychological studies related to work commitment. These are four dimensions in which commitment is indicated by support, recognition, learning and evaluation. That is to say, the labor commitment can be observed from the support that the organization gives to the individual for their personal, family and work development. In this sense, the leaders of the organization that recognize specific actions to the individual encourage and recognition of the labor, family or international groups in which the individual is inserted. In this way, a concrete action that implies specific recognition supposes specific learning as a symptom of work commitment. Finally, in the case of organizations with social commitment and that transfer these values to individuals, they generate a series of personal values that can be observed as symptoms of their work commitment.

The analysis of covariances shows negative and positive, significant and spurious associations between the indicators of labor commitment [20]. In the case of the commitment that the surveyed sample has with the institution in which they work, it is related to the commitment of growth in couples. Measure that institutional objectives are go with some couple shared goals. In this sense, interpersonal dynamics could be interrelated with other collaborative dynamics that in the workplace are inherent in the task climate or the relationship climate. In contrast, selfcommitment, which reflects a propensity for individualism in reference to institutional commitment, which implies a propensity for collectivism, are opposite indicators since while the values of a variable increase, a decrease in the values of the other variable [21]. This is that personal purposes do not seem to converge with the interests of the institution where Social Work professionals carry out their functions. In the case of the supraordinal indicator which denotes a commitment beyond the simple functions of observation, interview, systematization, and intervention that requires profession, criticism and Stas functions is linked with interpersonal goals [22]. In this sense, the task climate and the relationship climate are closer to the profession's critical commitment than to the conflict, since the questioning of the assistance functions is 
necessary in the development of Social Work. Maybe it by reason that criticism of the profession had a negative relationship with the healthcare commitment.

On the other hand, the care commitment had its greatest link with the family commitment [23]. Both indicators maintained positive relationships from which it is possible to deduce that the surveyed sample shows a close relationship between the practice of the profession as a healthcare commitment and the type of group to which they belong or want to belong. It is a third socialization of the social work professional in which public policies and welfare programs seem to complement the second order socialization of the families and groups that surrounded the Social Work professionals in their development. On the contrary, the assistance commitment is negatively related to the interpersonal commitment [24]. Despite the fact that the relationship is spurious, other variables influence both commitments, such an association is relevant since the commitment to the institution that projects and directs social programs fades before the interrelation of the Social Work professional with other colleagues, groups vulnerable, reference or belonging. Commitment to the closer interpersonal circle is opposed to institutional policy. For the relationship between engagement and collaborative family engagement is possible to note that the primary socialization group serves as a supplement to the group socialization second order. In systemic terms, the mesosystem in which family and work are indicators par excellence can be explained from commitment as a multidimensional factor. Not, however, collaborative commitment is negatively related to interpersonal engagement.

On the other hand, commitment to oneself is positively associated with commitment to the profession: social work [25]. The s identity is personal and labor to be linked explain the consistent practice of the profession even despite the vicissitudes of it. In a context in which the functions of social work are reduced to technical procedures and risk inherent to socio - economic studies, professional social work manifest a to association close between the functions of your l work your personal goals $d$ and life, but such Aspects are overshadowed by relationships with colleagues as profession commitment is opposite to interpersonal commitment. It is worth noting the association between institutional and supraordinal commitment assumes that the rules of health centers and the recognition of bosses towards the professional practice of social workers are associated in such a way that the granting of resources, socioeconomic studies or home visits seem to be linked to the organizational structure in terms of the distribution of resources or microcredits [6]. In the case of personal commitment and that derived from family recognition, the relationship suggests an interlocution between the information that the family members have on Social Work and their professional practice. This finding is relevant considering model, since it confirms the hypothesis that commitment is the result of organizational factors in reference to personal desires.
Once the covariances between the indicators were established, a reflective model was estimated [26]. A greater correlation between the labor-organizational commitment and institutional indicator. In this sense, the psychological construct of the workorganizational commitment is explained by the commitment to institutional policy. Apparently, Social Work professionals are influenced by organizational principles rather than by the personal, interpersonal, collaborative, functional, professional, healthcare, family or critical objectives inherent in Social Work.

In an opposite sense, the critical commitment of the profession was negatively related to the factor [27]. Such result complements the assumption around which Social Work professionals adjust their objectives to the internal policies of the institution for which they work, although they coexist with other principles that guide organizational commitment. Finally, the adjustment and residual indices were estimated to test the hypothesis regarding the configuration of an organizational commitment that would have as indicators aspects inherent to the individual, family, colleagues, functions, policies and structure in which each social worker is inserted. The results show that the null hypothesis can be accepted since the adjustment indices are close to unity and the residual close to zero. In summary, studies of work commitment show a multidimensionality of factors that would explain the emergence of a favorable disposition to risk events and contingent situations. It is an organizational response to excessive demands regarding the resources and capacities available in health institutions.

\section{Modelling variables and dimensions of labor commitment}

From the theoretical, conceptual and empirical frameworks reviewed, it is possible to model the variables and dimensions related to work commitment, considering four axes of discussion predictive of work performance (Figure 1).

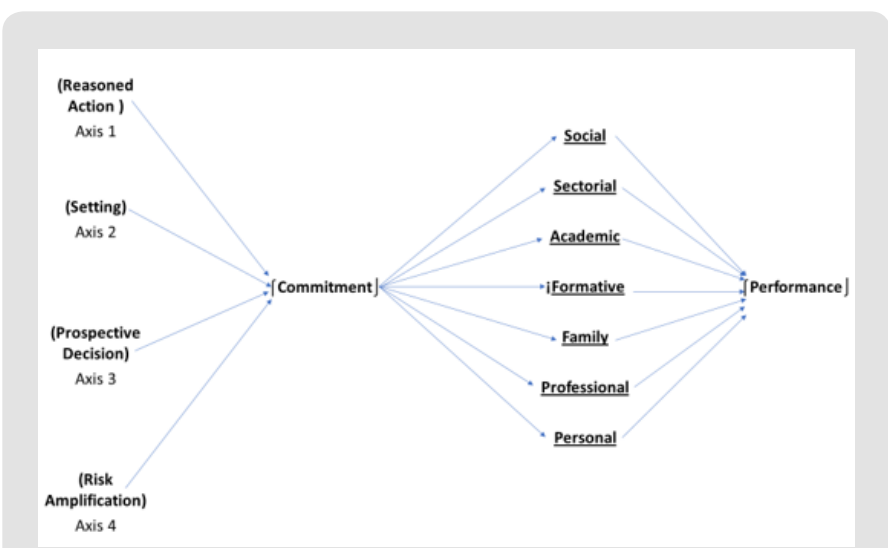

Figure 1: Modelling variables and dimensions of labor commitment.

Note: Elaborated with literature review; $($ ) = Dimensional axis, $\lceil$ J = Factorial Construct ___ = Latent variable.

Axis 1: Explanation of performance from reasoned action through the seven dimensions of labor commitment [28]. The 
empirical evidence indicates that reasoned action highlights an academic commitment that would explain, through favorable health provisions, a job performance in non-contingent or risky situations, but if this were the case, a spontaneous processing would emerge that would activate emotional dispositions which support a family or educational commitment due to the influence of people close to the health professional with their reasoned actions in their health functions.

Axis 2: Explanation of performance from setting through the seven dimensions of labor commitment [29]. The establishment of a health policy reflects a public, political and scientific agenda in which academic, training and professional commitment are involved. These are positive provisions for health promotion, as well as disease prevention, albeit motivated by speeches or narratives of union leaders by health professionals. This is the case of those who mobilize with the purpose of demanding better conditions for their work, as well as those who participate in debate forums about the vicissitudes or blunders of the authorities in the matter. The transfer of knowledge is the goal of those who have this profile of commitment to their colleagues and users of the services, but disseminators in the media.

Axis 3: Explanation of performance from prospective decision through the seven dimensions of labor commitment [11]. Doctors without borders represent this axis because they calculate high costs but also maximum benefits when exercising their profession in unhealthy, risky, threatening, contingent and uncertain conditions. Resource scarcity and management are hallmarks of this commitment profile which encompasses everyone except the family. These are liberal dimensions of commitment that do not match the traditional dimension of the family, which is a comfort zone.

Axis 4: Explanation of performance from amplification of risk through the seven dimensions of labor commitment [11]. Health professionals who attend unexpected calls without a selection system can contribute to the amplification of risk if it is a health crisis event such as an epidemic or a pandemic. In one sense, general amplifying risk assumes that healthcare is no longer seen as a solution but as an extension of the problem. The prestige and reputation of a health system is based on a commitment to society in general and to the vulnerable, marginalized or excluded sectors. Even personal commitment is forged from that institutional prestige that can be everted in the face of increased lethality.

In summary, work commitment, its explanatory conceptual theoretical frameworks and its dimensions of analysis reflect a health system focused on intellectual capital that studies have revealed as a factor of reputation and prestige, but also of risk amplification. When the system enjoys a good reputation, even the prospective decisions of its members are viewed favorably, as is the case of doctors without borders or the red cross, but on the contrary, they are assumed to be part of the problem in a pandemic with a high fatality rate. Such a process is exacerbated when the media enters the scene. This is the case of reports in health centers overwhelmed by the rate of infections, illnesses and deaths.

\section{Method}

Design. An exploratory, cross-sectional and correlational study was carried out.

\section{Sample}

Non-probability selection of 100 professionals (50 women and 50 men with an average age of 33 years and 7 having graduated) from Social Work in health centers in the state of Morelos (Mexico) with an average monthly income of 870 USD (DE $=12,5$ USD) seven years average work experience ( $\mathrm{DE}=2,3$ ). Organizational commitment is influenced by social policy and assistance program was considered suitable as a criterion for inclusion and 1 know the Plan Development National and Institutional and professional practice areas.

\section{Instrument}

Questions related to sex, age, income, origin, experience and civil and family situation were included. Used the Scale Commitment Labor which includes reagents around perceptions of items

\section{Procedure}

Respondents their commitment regarding specific situations of their job functions and organizational environment. Through a telephone contact with the selected sample in which an interview was requested and whose purposes would be merely academic and institutional to follow up on the graduates, whether they were graduates or not. Once the appointment was established, a questionnaire was provided in which the socio- demographic, economic and psychological or organizational questions were included. In cases where there was a tendency to the same response option or, the lack of response, they were asked to write on the back the reasons why they answered with the same response option or, if applicable, the absence of them. Data were captured in the Statistical Program for Social Sciences (SPSS) and structural equation analyzes were estimated with the help of the Structural Moment Analysis (AMOS) program and the Relationships program Linear Structural (LISREL).

\section{Analysis}

The establishment of the structural model of reflective relationships between work commitment and its indicators was carried out considering the normality, reliability and validity of the scale measured by the psychological construct. The kurtosis parameter was used to establish the normality of the distribution of responses to the level of commitment questioned. The results show that the kurtosis parameter had a value of less than eight, which is the minimum suggested to assume normal distribution. In the case of reliability, the Cronbach's alpha value allowed establishing 
the relationship between each question and the scale. The value exceeding 0, 60 was considered as evidence of internal consistency. Finally, the exploratory factor analysis of principal components and varimax rotation in which the factor weights greater than 0,300 allowed us to deduce the emergence of the commitment from eight indicators. Once normality, reliability and validity were established, the covariances between the indicators were established to model the existing relationships with the organizational factor.

\section{Results}

Table 1 shows the descriptive values that allow multivariate analyzes such as reliability, adequacy, sphericity and validity in order to appreciate the factor structure of work commitment.
The factorial structure made up of seven dimensions related to the social, sectoral, academic, training, family, professional and personal aspects explained $53 \%$ of the total variance, suggesting the extension of work to other dimensions that the literature identifies as antecedents of social work; charity, assistance or altruism. The social factor explained the highest percentage of the variance with $16 \%$ indicating that the labor commitment of the surveyed sample to be linked to the reputation, prestige and image of the institution vis-à-vis society in general. This is an in-depth commitment to local values, norms, uses and customs that reflect a demand and demand for attention from health professionals, even more in the face of risky events or contingent situations.

Table 1: Instrument description.

\begin{tabular}{|c|c|c|c|c|c|c|c|c|c|c|}
\hline $\mathbf{R}$ & M & SD & A & F1 & F2 & F3 & F4 & F5 & F6 & F7 \\
\hline$r 1$ & 4,10 & 1,01 & 0,781 & 0,562 & & & & & & \\
\hline$r 2$ & 4,35 & 1,02 & 0,793 & 0,671 & & & & & & \\
\hline$r 3$ & 4,61 & 1,04 & 0,793 & 0,632 & & & & & & \\
\hline$r 4$ & 4,30 & 1,04 & 0,763 & 0,651 & & & & & & \\
\hline$r 5$ & 4,03 & 1,05 & 0,761 & & 0,561 & & & & & \\
\hline$r 6$ & 4,01 & 1,54 & 0,703 & & 0,672 & & & & & \\
\hline$r 7$ & 4,76 & 1,57 & 0,794 & & 0,681 & & & & & \\
\hline$r 8$ & 4,19 & 1,03 & 0,704 & & 0,690 & & & & & \\
\hline$r 9$ & 4,10 & 1,24 & 0,761 & & & 0,651 & & & & \\
\hline$r 10$ & 4,15 & 1,35 & 0,783 & & & 0,572 & & & & \\
\hline$r 11$ & 4,12 & 1,46 & 0,762 & & & 0,498 & & & & \\
\hline$r 12$ & 4,16 & 1,81 & 0,751 & & & 0,561 & & & & \\
\hline$r 13$ & 4,95 & 1,43 & 0,793 & & & & 0,561 & & & \\
\hline$r 14$ & 4,65 & 1,01 & 0,764 & & & & 0,672 & & & \\
\hline$r 15$ & 4,62 & 1,36 & 0,712 & & & & 0,461 & & & \\
\hline$r 16$ & 4,34 & 1,37 & 0,761 & & & & 0,490 & & & \\
\hline$r 17$ & 4,21 & 1,29 & 0,719 & & & & & 0,671 & & \\
\hline$r 18$ & 4,35 & 1,42 & 0,715 & & & & & 0,580 & & \\
\hline$r 19$ & 4,23 & 1,47 & 0,719 & & & & & 0,572 & & \\
\hline$r 20$ & 4,13 & 1,58 & 0,762 & & & & & 0,591 & & \\
\hline$r 21$ & 4,32 & 1,31 & 0,716 & & & & & & 0,603 & \\
\hline$r 22$ & 4,33 & 1,43 & 0,782 & & & & & & 0,692 & \\
\hline$r 23$ & 4,27 & 1,54 & 0,763 & & & & & & 0,687 & \\
\hline$r 24$ & 4,10 & 1,65 & 0,701 & & & & & & 0,609 & \\
\hline$r 25$ & 4,05 & 1,39 & 0,761 & & & & & & & 0,61 \\
\hline$r 26$ & 4,02 & 1,67 & 0,732 & & & & & & & 0,572 \\
\hline$r 27$ & 4,28 & 1,25 & 0,712 & & & & & & & 0,503 \\
\hline$r 28$ & 4,04 & 1,47 & 0,742 & & & & & & & 0,601 \\
\hline
\end{tabular}

Note: Elaborated with data study; $\mathrm{R}=$ Reactive, $\mathrm{M}=$ Mean, $\mathrm{SD}=$ Standard Deviation, $\mathrm{A}=$ Alpha excluded value item. Adequation and Sphericity $\left\{\chi^{2}=21,23(12 \mathrm{df}) \mathrm{p}<, 05 ; \mathrm{KMO}=, 0627 \int \mathrm{F} 1=\right.$ Social $(16 \%$ total variance explained and alpha with 0,778$), \mathrm{F} 2=\mathrm{Sectorial}$ (13\% total variance explained and alpha with 0,765$), \mathrm{F} 3=$ Academic $(10 \%$ total variance explained and alpha with 0,780$), \mathrm{F} 4=$ Formative (7\% total variance explained and alpha with 0,756), F5 = family (4\% total variance explained and alpha with 0,752$)$, F6 = Professional ( $2 \%$ total variance explained and alpha with 0,790$),$ F7 = Personal ( $1 \%$ total variance explained and alpha with 0,760$)$. All items are answered with one of five options ranging from $0=$ "not at all likely" to $5=$ "quite likely". 


\section{Discussion}

The contribution of the present work to the state of the question lies in the validity of an instrument that measures seven dimensions related to work commitment which explained $53 \%$ of the total variance, although the results are limited to the study sample, suggest the extension from the proposal to other risk scenarios and contingent situations in order to confirm the structure of provisions and its seven factors. In relation to the theoretical and conceptual frameworks that highlight four axes of discussion around the commitment to the workplace, this work has shown that the social factor prevails over other areas, suggesting the systematic observation of its indicators in light of the theory of forward-looking decisions and amplification of risk [30-32]. The anticipation of haphazard and uncertain scenarios will allow the generation of didactic sequences aimed at the formation of intellectual capital to respond efficiently, effectively and effectively to the health demands of an eventual crisis. About studies of work commitment in which multiple dimensions stand out, this work confirms those findings reported in the literature, but suggests the observation of other dimensions inherent in social work such as charity, altruism and limited assistance. These results allow the design of virtual pedagogical sequences in which the training of professionals is established from scientific protocols rather than from interventional experience. In relation to the modeling of variables, the present study has demonstrated the emergence of a factorial structure of main axes with promax rotation in which it was possible to observe the concomitance of factors and indicators. Such an exercise will allow the design of interventions based on the social factor in order to take advantage of the prestige, reputation and image of an institution in the face of the pandemic.

In summary, the literature consulted highlighting the multidimensionality of work commitment seems to show that it is a stable structure as the responsibilities of health professionals diversify, even when they can amplify risks, they are scenarios of great public confidence to face a health crisis like COVID-19

\section{Conclusion}

The present study has established seven dimensions of organizational commitment to show the differences between the commitment derived from a social, sectorial, academic, formative, family, professional and personal. The convergence between these principles of contextual localization and identity in the face of power relations allows us to deduce that work commitment is a network of perceptions, beliefs, attitudes, decisions and actions aimed at the interrelation between the eight wielded dimensions. However, the prevalence of social commitment seems to show that Social Work professionals adjust their objectives to the structure and policy of the organization for which they work. Such findings are relevant considering the theory`s since they complement one of its principles related to customer service. To the extent that organizations follow a policy of evaluation and satisfaction of service quality, they foster an increase in the responsibilities, expectations and commitments of their employees. Thus, this study has found that adjusting to an organization's norms and policies prevails over personal, interpersonal, collaborative, and family goals. That is, if in the institutions where Social Work professionals, service quality policies were followed and productivity was established from consecutive evaluations, the surveyed sample would adjust its primary commitments to the objectives of said institution [33-35].

However, the command structure, according to the studies show that leadership style explains a higher percentage of the variance of work commitment in reference to the satisfaction of life, performance or productivity. In this sense, this research argues that commitment to the institution by coexisting with personal commitment, explains the influence of leadership style. In the context of the study, the surveyed sample manifested a work commitment consisting of matching their expectations with the mission and vision of the institution where they work. Therefore, it is logical to think that the leadership style complements the primary socialization.

Despite the contributions made, it is recommended to extend the study to leadership styles to develop a theory that allows explaining the influence of the institutional power structure on the labor commitment of Social Work professionals. If it is considered that in the institutions the leadership is occupied by other health professionals such as administrators, accountants or doctors, then it would be pertinent to explain the areas of opportunity for Social Work professionals when assuming a greater commitment and responsibility: planning an institution. Now, regarding the construction of an explanatory model of job satisfaction as an indicator of efficiency in the professional practice of Social Work, it is necessary to consider the incidence of social programs and sector strategies, since the dynamics of evaluating social and institutional policies it involves achieving objectives by a human capital willing to reproduce development plans on social needs as well as managing opportunities and capacities to spread responsibilities such as health in vulnerable, marginalized or excluded sectors. The success of development policies is centered on the level of commitment of those who carry out institutional plans and strategies, manage resources and promote a culture of self-care without which any development plan would be fallible.

In Mexico, social policies and programs involve assisting vulnerable, marginalized or excluded groups through the professional practice of Social Work. In the health area, management and promotion are areas of intervention of Social Work. In other words, as the State encourages human development in its spheres of health, education and employment, it affects institutional strategies for the prevention and treatment of diseases. However, the institutional political machinery of the health sector requires 
talents committed to low-income social groups, since among these networks for local development will be formed. For this reason, it is relevant to study the indicators of work commitment in professionals linked to social assistance programs. A diagnosis of the areas of opportunity and the virtues of the health promotion and management system will allow us to discuss the emergence of new paradigms of social assistance focused on promoters of human development.

Even though public institutions are limited to a National Development Plan, the professional practice of Social Work is often conditioned by local, institutional situations or by the work climate, salary or stress that personalized attention implies. In this sense, it is necessary to establish the dimensions of the organizational commitment of the Social Work professionals, since a high degree of commitment suggests an efficient level of attention which could translate into a favorable evaluation of social policies and institutional programs. For the health sector, labor commitment to being associated with the management and health promotion, is set by indicators that are responsible for the structures and policies extol institutional predicting personal actions. In this sense, the work commitment has been a transmitter relations and conflicts to the interior of an institution that inhibit or potentiate job satisfaction. As professional commitment transfers organizational values of collaborative interrelation, it affects the expectations of the members of an organization. Such a process supposes the coexistence between indicators of labor commitment that by their nature are complementary and opposite. Ie organizational commitment contains two processes of socialization, first allusive order earlier that place the individual in a context and second order relative earlier that identify a structure of power relations, both can be complementary or opposite.

In the field of Social Work, institutions function as secondorder socialization structures to influence the perceptions, beliefs, attitudes, decisions and actions of their employees. In principle, institutions are a scenario of political rationality in which the State uses social assistance programs that Social Work professionals must follow and, in any case, perfect said system to achieve the favorable evaluation of public policies and social programs. Such a process of political rationality can be complementary or antagonistic to the principles that guide personal, interpersonal, family or collaborative commitment in the employees of a public institution. For this reason, it is necessary to clarify the dimensions of organizational commitment based on the complexity that the exercise of Social Work implies. In this sense, the present study seeks to establish the dimensions of work commitment considering levels of institutional complexity that would frame the disagreement with indicators of a commitment derived from primary socialization.

\section{References}

1. Cruz García Lirios (2020) Models of fixed effects of diffuse variables in the formation of intellectual capital. International Journal of Engineering Research and Development 15(9): 1-7.
2. García C, Bustos J M, Carreón J, Hernández J (2017) Theoretical and conceptual frameworks around local development. Margin 85: 1-11.

3. García C, Espinoza F, Carreón J (2018) Model of intangible assets and capitals in organizations. International Journal of Research in Humanities and Social Studies 5(4): 1-12.

4. Hernandez J, Margarita Juarez Najera, Hector Daniel Molina Ruiz, Michiko Amemiya Ramirez, Cruz Garcia Lirios, et al. (2020) Specification of a social intervention model against covid-19. Biochemical Journal of Scientific Technical Research 26(4): 1-4.

5. Juárez M (2020) Specification a model for study of corporate assistance. Global Journal of Archeology \& Anthropology 11(2): 50-54.

6. Bermudez G, Rigoberto Sanchez Rosales, Francisco Espinoza-Morales, Enrique Martinez Munoz, Arturo Sanchez-Sanchez (2019) Metaanalytical validity of the social entrepreneurship inventory: A study of random effects size. Global Journal of Management \& Business research 19(10): 15-19.

7. Boils B (2020) Specification a model of entrepreneurship: from opportunism to innovation. International Journal of Advances Research 8(3): 20-24.

8. Carreon J (2019) Model of the determinants of human capital. International Journal of Advances in Social Science and Humanities, $7(8): 1-5$

9. Garcia C (2020) Specification of a model for study of the utility entrepreneurship. Global Journal of Human Social Science 20(1): 35-38.

10. Hernández TJ, Carreón J, García C (2019) Netizens Millennials. International Journal of Advances Engineering Research Science 6(7): $1-5$

11. Villegas E, Carreón J, García C, S Clark (2019) Specification a model for study of intellectual capital. International Journal of Economics and Management Studies 10: 1-2.

12. Elizarraráz G, Molina H D, Quintero M L, Sánchez R, García C (2018) Discourses on organizational lucidity in strategic alliances and knowledge networks among coffee-growing MSMEs in central Mexico. Margen 89: 1-11.

13. Fierro E, Alvarado M G, Garcia C (2018) Contrast a model of labor commitment in a public institution of the center of Mexico. Psichology $7(13): 32-48$

14. Garcia C (2019) Dimensions of human development theory. Ehquidad 11: $27-54$.

15. Garcia C, Martinez E, Sanchez A (2018) Exploratory factorial structure of the institutional dimensions of work commitment in a higher education institution (IES) in central Mexico. Perspectivas 20(2): 65-87.

16. García C, Juárez M, Bustos JM (2018) Specification of a model for the study of local governance. Synchrony 22(73): 459-472.

17. Garcia J J, Delgado M A, Y Garcia C (2018) Reliability And Validity Of An Instrument That Measures Health Wellness. Eureka 15(82): 44-52.

18. Hernández J, Anguiano F, Valdés O, Limón G A, García C (2018) Reliability and validity of a scale that measures vocational training expectations. Margen 89: 1-13.

19. Hernández J, Carreón J, Bustos JM, García C (2018) Organizational cyberculture model in knowledge innovation. Management vision 18(2): 235-253.

20. Juarez M, Garcia C, Quintero M L (2019) Confirmatory factor composition of the perceived labor norm. Ciencias Sociales 1: 1-14.

21. Llamas B, Lopez S, Garcia C (2019) Specification of a model of adherence to treatment. Ajayu 17(1): 140-150.

22. Martínez E, Espinoza F, García C (2019) Models of the determinants of vocational training. International Journal in Advances of Social Science and Humanities 6(7): 1-5. 
23. Moreno E, Ferrusca FJR, Balderas WIA, Vázquez FRS, Bello JM, et al. (2019) Governance of social representations of quality of life. Psychological Research International Journal. 4(1): 1-5.

24. Quiroz C Y (2019) Specification a model of culture knowledge management. Global Advances Research Journal of Agricultural Science 8(10): 1-4.

25. Sánchez A, Hernández T J, Martínez E, Villegas E, García C (2018) Organizational culture in activating micro-enterprises of local development. Margen 89: 1-10.

26. Villegas E (2018) Governance of intellectual capital millennials for the creation of intangible organizational value. Net Journal Social Science 6(1): $1-9$

27. Sánchez A, Hernández TJ, Quintero ML, Espinoza F, García C (2018) Networks and knowledge around the learning of educational organizational complexity. Hologram 15(28): 223-253.

28. Soto A, Dorner A, Garcia C, Hernández TJ (2018) The collective welfare as a topic of family re-socialization in the society of informational capitalism. Utopia and Latin American Praxis 23(83): 52-56.

29. Veliz A, Carreon J, Dorner A, Estay JG, Garcia C (2018) Democracy, governance and ethical behaviors: transversal axes of training. Option 34(86): 152-175

ISSN: 2574-1241

DOI: $10.26717 /$ BJSTR.2020.31.005134

Cruz García Lirios. Biomed J Sci \& Tech Res

CC (i) This work is licensed under Creative

Submission Link: https://biomedres.us/submit-manuscript.php
30. García C, Martínez E, Quintero M L (2019) Exploratory factorial structure climate and labor flexibility. Turns 20(2): 55-72.

31. Cruz García Lirios (2019) Categorical exploratory structure of intellectual capital formation in its phase of intangible organizational assets. Journal of Social Science Research 6(8): 1-6.

32. Cruz García Lirios (2019) Exploratory categorical structure of employment expectations. Journal of Social Science Research 6(8): 1-6.

33. Fierro E, Nava S, Garcia C (2018) Reliability And Validity Of An Instrument That Measures Organizational Commitment In A Community Health Center. Tlatemoani 29: 42-68.

34. Cruz García Lirios, Enrique Martínez Muñoz, María Luisa Quintero Soto (2018) Exploratory factorial structure climate and labour flexibility. Viarjes 20(2): 55-72.

35. Hernández Valdés, García Lirios, Carreón Guillén (2019) Specification a model for study of entrepreneurship in household heads. International Journal of Social Science and Education 6(8): 1-5.

$\begin{array}{ll}\text { BIOMEDICAL } & \text { Assets of Publishing with us } \\ \text { RESEARCHES } & \text { - Global archiving of articles } \\ & \text { - Immediate, unrestricted online access } \\ & \text { - Rigorous Peer Review Process } \\ & \end{array}$

